\title{
Tetraspanin 3, as a Novel Regulator of Intracellular B1 Integrin Recycling, Promotes Non-Small Cell Lung Cancer Cell Proliferation
}

Hongbo Su

China Medical University

Yitong Xu

China Medical University

Yao Zhang

China Medical University

Xizi Jiang

China Medical University

Qingfu Zhang

China Medical University

Hongjiu Ren

China Medical University

Zifang Zou

China Medical University

Xueshan Qiu ( $\sim$ xsqiu@cmu.edu.cn )

China Medical University https://orcid.org/0000-0001-9481-8249

\section{Research}

Keywords: beta 1 integrin, tetraspanin 3, non-small cell lung carcinoma, Rab11a

Posted Date: June 9th, 2021

DOI: https://doi.org/10.21203/rs.3.rs-572884/v1

License: (1) (1) This work is licensed under a Creative Commons Attribution 4.0 International License. Read Full License 


\section{Abstract}

Background: The role of tetraspanins in cancer development has been widely reported. However, the expression and roles of tetraspanin 3 (TSPAN3) in solid tumors, including non-small cell lung cancer (NSCLC), have not yet been extensively investigated. In this study, we explored the role of TSPAN3 in NSCLC and its potential role in the trafficking of integrin $\beta 1$, which is highly expressed in this type of cancer.

Methods: The expression of TSPAN3 in NSCLC tissue and NSCLC cell lines was evaluated, and the correlation between TSPAN3 expression and disease progression was assessed. Furthermore, loss- and gain-of-expression studies were conducted to determine the biological function of TSPAN3 both in vivo and vitro. The regulatory role of TSPAN3 in $\beta 1$ integrin expression and intracellular recycling was studied through western blot, RT-PCR, and immunofluorescence analyses.

Results: TSPAN3 was found to be highly expressed in lung cancer cells and tissues. Moreover, high levels of TSPAN3 positively correlated with poor differentiation, lymph node involvement, advanced pathological tumor-node-metastasis stage, and poor prognosis in NSCLC patients. TSPAN3 showed the potential to promote the proliferation of NSCLC cells in vitro and in vivo. Specifically, TSPAN3 was found to interact with $\beta 1$ integrin and Rab11a, thereby facilitating the sorting of $\beta 1$ integrin into Rab11a endosomes and promoting $\beta 1$ integrin recycling and upregulation.

Conclusions: These findings reveal a novel role for TSPAN3 in the regulation of intracellular recycling of $\beta 1$ integrin. Hence, TSPAN3 may represent a potentially valuable therapeutic target for NSCLC.

\section{Background}

The trafficking of integrins through the endosomal pathway is known to influence their function [1], and it plays a crucial role in cancer progression; thus, it should be considered when assessing the efficacy of potential anticancer agents [2].

Surface integrins are continuously endocytosed and efficiently recycled back to the cell membrane $[3,4]$. Previous studies have shown that integrins are transported to the early endosomes after endocytosis, where sorting decisions are made as to whether the endocytosed integrins will be transferred to late endosomes and lysosomes for degradation, or they will be recycled through one of two distinct routes. Instead of being degraded, most integrins return to the cell membrane through the Ras-related protein Rab4mediated route (also called the short loop) or move to the perinuclear recycling compartment and are transported to the cell membrane through the Rab11-mediated route (also called the long loop) [4-7]. Although the understanding of integrin trafficking is rapidly expanding, the mechanism through which cells select a specific trafficking route for different integrins remains unclear. Importantly, the mediatory role of Rabs in the trafficking of specific integrins requires further investigation [8]. 
Tetraspanins are abundantly expressed in several tissues; however, their biological functions have not been studied extensively [9]. These proteins contain two extracellular domains, the large extracellular loop (LEL) domain and the small extracellular loop (SEL) domain. The LEL domain was reported to be crucial for the interaction of tetraspanins with multiple receptors and signaling molecules such as $\beta 1$ integrins. Certain tetraspanins, such as tetraspanin 3 (TSPAN3), harbor the YXXФ tyrosine-based sorting motif that has been widely reported to be associated with endocytosis or trafficking $[10,11]$. Additionally, a previous study has suggested that the YXXФ motif of $\beta 2$ integrins is essential for their recycling [12].

Accumulating evidence indicates the crucial role played by tetraspanins in regulating the function and trafficking of membrane proteins. For example, downregulation of tetraspanin CD151 significantly inhibits the endocytosis of a3 $\beta 1$ integrin in cells seeded on laminin-5 [13]. Similarly, the expression of surface CD19 has been reported to be downregulated in $\mathrm{CD} 81^{-}$cells, suggesting a regulatory role for CD81 in CD19 trafficking. Further analysis suggested that the transport rate of CD19 from the endoplasmic reticulum to the Golgi compartment was significantly slower in $\mathrm{CD} 81^{-}$cells than in $\mathrm{CD} 81^{+}$ cells $[14,15]$.

TSPAN3 belongs to the tetraspanin superfamily. The functions of TSPAN3 have not been well investigated until recently and have only been studied in neural cells and acute myeloid leukemia [16]. TSPAN3 was reported to form a complex with oligodendrocyte-specific protein (OSP; also known as claudin-11) and $\beta 1$ integrin in oligodendrocytes. Moreover, overexpression of TSPAN3 was reported to promote the proliferation of mouse oligodendrocyte cells $[17,18]$.

This study aimed to explore TSPAN3 expression and clinicopathological significance in non-small cell lung carcinoma (NSCLC) to determine its potential role. Additionally, based on previous research, the study further aimed to validate the role of TSPAN3 in intracellular trafficking and explore the regulatory mechanism of TSPAN3 on $\beta 1$ integrin in NSCLC.

\section{Methods}

\section{Patients and specimens}

Tissue samples from 105 patients diagnosed with NSCLC between 2013 and 2015, and corresponding clinicopathological information, were acquired from the Pathology Department of the First Affiliated Hospital of China Medical University. All tumors were collected through curative surgical resection. None of the enrolled patients had received pre-surgical chemotherapy or radiation. Among the 105 tissue samples, 81 were paraffin-embedded tissues that were used for immunohistochemistry analysis, whereas 24 tissues were fresh samples, of which eight and 16 samples were used for western blotting and realtime polymerase chain reaction (RT-PCR) analyses, respectively. All patients were included in the study retrospectively and randomly. The study was approved by the Medical Research Ethics Committee of the First Affiliated Hospital of China Medical University (KLS [2020] No. 2020-40-2, Shenyang China). The requirement for informed consent was waivered considering the retrospective nature of the study. All 81 
patients whose samples were used for immunohistochemistry analysis were followed up for at least 3 years.

\section{Cell lines and cell culture}

The human bronchial epithelial (HBE) cell line was obtained from the American Type Culture Collection (ATCC; Manassas, VA, USA). The other cell lines (A549, H460, H1299, H1975, H226, H661, 293T, and SKMES-1) used were purchased from the Shanghai Cell Bank (Shanghai, China). A549, H1299, H460, H661, H226, and H1975 cells were cultured in RPMI-1640 medium, SK-MES-1 cells were cultured in minimal essential medium, and HBE and 293T cells were cultured in Dulbecco's modified Eagle's medium. Except for the media used for the $\mathrm{H} 1975$ cell line, all other media were supplemented with $10 \%$ fetal bovine serum (FBS). The media for the H1975 cell line was supplemented with $20 \%$ FBS. All media were purchased from Gibco (Waltham, MA, USA); FBS was purchased from Clark Bioscience (Webster, TX, USA).

\section{Immunohistochemistry (IHC)}

As described previously [19], tissue sections were probed with appropriate primary antibodies (Additional file 1). Staining intensity was scored as follows: 0 (no staining), 1 (weak), 2 (moderate), or 3 (high). Percentage scores were assigned as follows: 1 (0-25\%), 2 (26-50\%), 3 (51-75\%), and 4 (76-100\%). The final score for each specimen (0-12) was calculated by multiplying the intensity score with the percentage score. Tumor tissues with scores $>6$ were considered to have positive expression, whereas those with scores $\leq 6$ were considered to have negative expression.

\section{Cell treatment and transfection}

TSPAN3- and RAB11a-specific siRNAs and scrambled control siRNAs were purchased from RiboBio (Guangzhou, China). The pCMV6-Myc-FLAG-TSPAN3 construct (\#RC203876) and the empty vector were purchased from OriGene (Rockville, MD, USA). The pCMV6-GFP-TSPAN3- $\triangle$ LEL construct containing a splice variant (LEL deletion) of wild-type TSPAN3 was purchased from TSINGKE Biological Technology (Beijing, China). Lipofectamine 3000 (Invitrogen, Waltham, MA, USA) was used for transfection according to the manufacturer's instructions.

Nocodazole (NZ) washout was performed to explore $\beta 1$ integrin trafficking as previously described [20]. After $12 \mathrm{~h}$ of serum starvation, A549 cells were treated with NZ (10 $\mu \mathrm{M}$; \#31430-18-9; MedChemExpress, Monmouth Junction, $\mathrm{NJ}, \mathrm{USA}$ ) for $4 \mathrm{~h}$ to completely depolymerize the microtubules (MTs). Then, the drug was washed off with serum-free medium to allow MT repolymerization at different intervals after NZ washout.

For fibronectin (FN) stimulation of cells, recombinant human FN (10 $\mu \mathrm{g} / \mathrm{mL}$; \#1918-FN-02M; R\&D Systems, Minneapolis, MN, USA) was added to RPMI-1640 medium supplemented with $1 \%$ FBS.

\section{Western blotting}


Whole-cell lysates were prepared from cells and tumor tissues using the NP-40 lysis buffer (P0013F, Beyotime Biotechnology, Shanghai, China) containing PMSF (1:100, ST506; Beyotime Biotechnology) and phosphatase inhibitor (1:100; B15002; Biotool, Shanghai, China). Total protein was quantified using the Bradford method, and proteins were resolved with $10 \%$ sodium dodecyl sulfate-polyacrylamide gel electrophoresis with the loading amount of $60 \mu \mathrm{g}$ and transferred onto polyvinylidene difluoride membranes (Millipore, Burlington, MA, USA). The membranes were then probed overnight with the appropriate primary antibodies at $4{ }^{\circ} \mathrm{C}$ (Additional files 1 and 2), washed three times with tris-buffered saline and Tween 20 (TBST) buffer, and incubated with horseradish peroxidase-conjugated anti-mouse or anti-rabbit secondary antibodies (1:2000; Proteintech) at about $22^{\circ} \mathrm{C}$ for $2 \mathrm{~h}$. Protein bands were visualized via enhanced chemiluminescence (H34080; Thermo Fisher Scientific) using a Biolmaging System (UVP, Upland, CA, USA). Relative expression was analyzed using ImageJ (National Institutes of Health, Washington, DC, USA); the expression of target proteins was normalized against that of GAPDH or $\beta$-actin.

\section{Quantitative RT-PCR}

Total RNA of cells and tissues were extracted as described previously [21]. Quantitative RT-PCR was carried out in a 7900HT Fast Real-time PCR System (Applied Biosystems, Foster City, CA, USA) using SYBR Premix Ex Taq II (RR820A, Takara Bio, Beijing, China) in a total volume of $20 \mu \mathrm{L}$ according to the manufacturer's instructions. Relative gene expression was calculated by the $2^{-\Delta \Delta C t}$ method; the $\beta$-actinencoding gene was used as the reference. The primer sequences used are listed as follows. All experiments were performed in triplicate.

TSPAN3 forward, 5'-ATGGAACCAACCCTGATGCTGCTAG-3';

TSPAN3 reverse, 5'-AGTCTCTCTGCAGCAGCTAAGAGGG-3';

ß1 integrin forward: 5'-CAAGAGAGCTGAAGACTATCCCA-3';

$\beta 1$ integrin reverse: 5'-TGAAGTCCGAAGTAATCCTCCT-3';

ß-actin forward: 5'-ATAGCACAGCCTGGATAGCAACGTAC-3';

ß-actin reverse: 5'-CACCTTCTACAATGAGCTGCGTGTG-3'.

\section{Cell proliferation assay}

Cells transfected with the TSPAN3-expressing plasmid or empty vector were seeded (3000 cells/well) in 96-well plates for MTS cell proliferation assay. Absorbance at $450 \mathrm{~nm}$ was detected every $24 \mathrm{~h}$. A growth curve was generated using the absorbance values after culture for 4 days.

\section{Colony formation assay}


Cells transfected with TSPAN3-expressing plasmid or empty vector were seeded (500 cells/well) in 6-well plates and cultured until the formation of visible colonies. Images were acquired on a Biolmaging System (DNR, Neve Yamin, Israel). All experiments were performed at least three times.

\section{Co-immunoprecipitation and mass spectrometric assay}

Assays were performed as previously described [22].

\section{Immunofluorescence}

The A549 and $\mathrm{H} 460$ cells $\left(2 \times 10^{5}\right)$ were seeded in 24-well plates and cultured for $24 \mathrm{~h}$, fixed with $4 \%$ paraformaldehyde for $10 \mathrm{~min}$, blocked with $5 \%$ bovine serum albumin for $2 \mathrm{~h}$, and then probed overnight with the appropriate primary antibodies at $4{ }^{\circ} \mathrm{C}$ (Additional file 1). After washing 3 times with PBS, the cells were incubated with TRITC-conjugated or FITC-conjugated secondary antibodies at approximately $22{ }^{\circ} \mathrm{C}$ for $2 \mathrm{~h}$, and the nuclei were stained with 4',6-diamidino-2-phenylindole (DAPl; C1005, Beyotime Biotechnology). Images were captured using an Olympus FV3000 laser-scanning confocal microscope (Olympus, Tokyo, Japan).

For quantification of $\beta 1$ integrin and Rab11a colocalization, the Pearson correlation coefficient was analyzed using the Fluoview software (FV31S-SW, Olympus, Tokyo, Japan).

\section{Flow cytometry}

A549 cells collected via trypsinization at different time points after NZ washout were washed with cold PBS. Resultant cells $\left(1 \times 10^{5}\right.$ cells/sample) were stained with PE-conjugated $\beta 1$ integrin antibody (1:100; \#12-0299-42; eBioscience, San Diego, CA, USA) at $4{ }^{\circ} \mathrm{C}$ for $20 \mathrm{~min}$. The samples were washed three times to remove unbound antibodies and fixed with cold $4 \%$ paraformaldehyde for $20 \mathrm{~min}$. The mean fluorescence intensity of each sample was measured using a FACScan flow cytometer (BD, Franklin Lakes, NJ, USA).

\section{Tumor formation in nude mice}

Nude mice used in this study were treated following the experimental animal ethics guidelines issued by the China Medical University. Four-week-old female BALB/c nude mice were purchased from Slac (Shanghai, China) and were maintained in a laminar-flow cabinet under specific pathogen-free conditions for one week before use. Each mouse was inoculated subcutaneously in the right axilla with $1 \times 107$ tumor cells (selected by G418) in $0.2 \mathrm{~mL}$ sterile phosphate-buffered saline. Four weeks after axilla inoculation, the mice were euthanized, and necropsies were performed to examine tumor growth.

\section{Statistical analyses}

The relationships between TSPAN3 expression and clinicopathological factors and $d \beta 1$ integrin were statistically analyzed and tested using the chi-squared test. The association between TSPAN3 expression 
and the prognosis of NSCLC patients was analyzed using both Kaplan-Meier and Cox proportional hazards models. All statistical analyses were performed using the SPSS 17.0 software (SPSS, Inc., Chicago, IL, USA). Differences between two groups were analyzed using a paired Student's t-test using Prism 6.0 (GraphPad Software, San Diego, CA, USA). P-values $<0.05$ were considered significant.

\section{Results}

\section{High expression of TSPAN3 in NSCLC predicts poor prognosis}

Immunohistochemistry and immunofluorescence analyses were performed to assess the expression of TSPAN3 in 81 NSCLC tissues and 2 NSCLC cell lines. TSPAN3 was found to be mainly localized in the cytoplasm. In addition, significantly higher levels of TSPAN3 were observed in NSCLC tissues than in the normal bronchial epithelium and submucosal glands (Figure 1a). Western blotting was performed to assess TSPAN3 expression in eight pairs of fresh NSCLC tissues and adjacent normal tissues. NSCLC specimens were found to express higher levels of TSPAN3 than their paired normal tissues (Figure 1b). Baseline expression of TSPAN3 was also examined in six human lung cancer cell lines as well as 293T, and HBE cell lines. TSPAN3 levels were higher in all six lung cancer cells than those in HBE cells (Figure 1c). High TSPAN3 expression in NSCLC was positively associated with poor differentiation, lymph node involvement, and advanced pathological tumor-node-metastasis stage $(P=0.006,0.006$, and 0.011 , respectively; Table 1) but showed no significant association with sex, age, or histological type $(P>0.05$; Table 1). Kaplan-Meier survival analysis after following up all 81 patients for at least 3 years further revealed that the survival period of patients with high TSPAN3 levels (1800.2 days, $95 \%$ confidence interval [CI]: 1595.3-2005.4) was significantly shorter than that of patients with low TSPAN3 levels (2072.8 days, 95\% Cl: 2029.8-2115.9) ( $P=0.014$; Figure 1e). Cox analyses also revealed that positive TSPAN3 expression $(P=0.043$; hazard ratio $=9.139,95 \% \mathrm{Cl}$ : $1.067-78.248$; Additional file 3$)$ was an independent prognostic factor in NSCLC.

\section{TSPAN3 interacts with $\beta 1$ integrin via its LEL domain and upregulates $\beta 1$ integrin expression in NSCLC cells}

Co-immunoprecipitation was performed in A549 cells to investigate whether TSPAN3 could interact with $\beta 1$ integrin. Both endogenous and transfected TSPAN3 showed efficient interaction with $\beta 1$ integrin (Figure $2 \mathrm{a}, \mathrm{b}$ ). As the LEL domain plays a vital role in mediating the interaction of TSPAN3 with other membrane proteins, a TSPAN3 LEL-deleted construct was generated (Figure 2c) to further explore the dependency of TSPAN3 and $\beta 1$-integrin interaction on the LEL domain. Co-immunoprecipitation revealed that the interaction between TSPAN3 and $\beta 1$-integrin was abrogated when the LEL domain was deleted (Figure 2d). Next, to investigate the regulation of $\beta 1$ integrin by TSPAN3, A549 and H460 cells (cells with moderate TSPAN3 expression) were transfected with a TSPAN3-expression vector. TSPAN3 overexpression upregulated $\beta 1$ integrin levels relative to the control, whereas TSPAN3 knockdown showed the opposite effect (Figure 2e). A549 and H460 cells were also transfected with plasmids harboring the wild-type or mutant (LEL-deleted; TSPAN3- $\triangle$ LEL) TSPAN3. Western blotting showed that overexpression 
of wild-type TSPAN3 resulted in upregulated expression of $\beta 1$ integrin, whereas that of the TSPAN3- $\triangle \mathrm{LEL}$ mutant had no such effect, which further suggested that LEL domain is required for the upregulation of $\beta 1$ integrin (Figure 2f).

We further validated the ability of TSPAN3 to upregulate the expression of $\beta 1$-integrin in 20 NSCLC samples that were randomly selected from the 81 tissues, through immunohistochemistry. Correlation analysis revealed that tissues with high levels of TSPAN3 also had high levels of $\beta 1$ integrin, whereas those with low levels of TSPAN3 had low levels of $\beta 1$ integrin (Figure $2 \mathrm{~g}$ ), indicating a positive correlation between the expression of TSPAN3 and $\beta 1$ integrin $(P=0.022$, Table 2$)$.

\section{TSPAN3 activates the FAK/MAPK pathway and promotes NSCLC cell proliferation via $\beta 1$ integrin}

Next, the effects of TSPAN3 overexpression and knockdown on the phosphorylation of the key intermediaries of the FAK/MAPK signaling pathway, which is downstream of $\beta 1$ integrin [23], were investigated. Overexpression of TSPAN3 resulted in significantly increased phosphorylation of FAK, MEK, and ERK, whereas overexpression of the LEL-deleted TSPAN3 mutant did not exhibit a similar effect, a finding that further demonstrated that TSPAN3 activates the FAK/MAPK signaling pathway through its interaction with $\beta 1$ integrin. Conversely, TSPAN3 knockdown resulted in reduced phosphorylation of these key factors (Figure 3a, b; Additional file 4).

The function of TSPAN3 in NSCLC cells was further explored. Colony formation and MTS assays revealed that TSPAN3 knockdown reduced the proliferation of A549 and H460 cells (Figure 3c, d), whereas overexpression of TSPAN3 in these cells failed to induce enhanced proliferation in both cell lines (Additional file 5). A549 and H460 cells were cultured in a liquid medium, which does not contain high levels of integrin ligands (similar to the extracellular matrix in vivo), which may explain why TSPAN3 overexpression failed to promote the proliferation of these cells. Therefore, A549 and H460 cells were treated with $\mathrm{FN}(10 \mu \mathrm{g} / \mathrm{mL})$, an integrin ligand. As expected, in the presence of FN, TSPAN3 overexpression promoted the proliferation of these NSCLC cells (Figure 3e). Furthermore, variations in TSPAN3 levels consistently affected the expression of proteins involved in cell proliferation. Specifically, the expression of cyclin D1 and cyclin B1 was upregulated following TSPAN3 overexpression, and overexpression of the TSPAN3- $\triangle$ LEL mutant abrogated these effects. Conversely, TSPAN3 knockdown decreased the expression of cyclin D1 and cyclin B1 (Figure 3b, f; Additional files 4 and 6).

To further explore the effects of TSPAN3 on NSCLC proliferation in vivo, a total of 10 nude mice were injected subcutaneously with $\mathrm{H} 1299$ cells stably transfected with the TSPAN3-overexpressing plasmid or the empty vector (selected by G418). Tumor formation rates in both groups were similar (4/5, 80\%). However, tumor volume and weight in the TSPAN3-overexpressing group were greater than those in the empty vector group (Figure 3g; Additional file 7). Taken together, these data suggest that TSPAN3 functions as a promoter of NSCLC progression in vitro and in vivo.

\section{TSPAN3 promotes $\beta 1$ integrin recycling in NSCLC cells}


To investigate the mechanism by which TSPAN3 regulates $\beta 1$ integrin production, $\beta 1$ integrin (ITGB1) mRNA levels were first measured. PCR analysis revealed that neither TSPAN3 overexpression nor its knockdown significantly affected ITGB1 expression (Figure 4a, b). Moreover, PCR analysis of 16 NSCLC samples showed no positive correlation between TSPAN3 and ITGB1 expression (Figure 4c), suggesting that TSPAN3 influences $\beta 1$ integrin production at the post-translational level.

Previous studies have reported that tetraspanins can regulate various aspects of membrane receptor trafficking. Thus, it is reasonable to hypothesize that TSPAN3 is involved in the regulation of $\beta 1$ integrin trafficking and recycling, thereby upregulating $\beta 1$ integrin expression. A previous study has shown that treatment of cells with NZ results in microtubular cytoskeleton disruption and ultimately $\beta 1$ integrin accumulation in focal adhesions, and upon NZ removal, the MTs re-polymerize, leading to the rapid endocytosis of $\beta 1$ integrin, allowing $\beta 1$ integrins to travel along the microtubules to Rab5-positive early endosomes and subsequently to the Rab11 compartment in the perinuclear region. Sixty minutes after NZ washout, most of the $\beta 1$ integrins were endocytosed, and surface $\beta 1$ integrin expression was reduced to a minimum. (Figure 4d) $[2,24,25]$. We validated these results in A549 cells, showing that 60 min after $\mathrm{NZ}$ washout, the surface $\beta 1$ integrin expression was about $50 \%$ of that at $0 \mathrm{~min}$, which showed the minimum level. Then, the surface $\beta 1$ integrin expression gradually recovered and almost returned to baseline levels 100 min after NZ washout (Figure 4e).

Thus, the NZ washout method was adopted to synchronize the endocytosis of $\beta 1$ integrins and further explore the impact of TSPAN3 on $\beta 1$ integrin recycling. Flow cytometry indicated that A549 cells lacking TSPAN3 could not restore the surface $\beta 1$ integrin levels $100 \mathrm{~min}$ after NZ washout (Figure 4f). Accordingly, knocking down TSPAN3 in A549 cells resulted in a failure in restoring FAK (Tyr397) phosphorylation to levels similar to those in the control group (100 min after NZ washout; Figure 4g). Immunofluorescence analysis of A549 cells was performed at different time points after NZ washout to verify the involvement of TSPAN3 in $\beta 1$ integrin recycling. At $60 \mathrm{~min}$, most of the $\beta 1$ integrin had been endocytosed and transported to the perinuclear region. At this time point, most of the TSPAN3 had localized to the perinuclear region and exhibited intense colocalization with $\beta 1$ integrin. At 120 min, most of the $\beta 1$ integrin had returned to the membrane, and the colocalization of TSPAN3 and $\beta 1$ integrin was lost (Figure 4h). Taken together, these data suggest that TSPAN3 is involved in, and promotes, the recycling of $\beta 1$ integrin.

\section{TSPAN3 promotes $\beta 1$ integrin recycling by facilitating its sorting into Rab11a recycling endosomes}

To further explore the specific mechanism underlying the involvement of TSPAN3 in $\beta 1$ integrin recycling, mass spectrometric analysis was performed. Consistent with reports that the Rab11-dependent mechanism is one of the major routes for integrin recycling, the mass spectrometric data (Additional files 8 and 9) indicated that TSPAN3 could promote $\beta 1$ integrin recycling via Rab11a. The interaction between TSPAN3 and Rab11a was further verified in A549 cells through co-immunoprecipitation (Figure 5a, b). Immunofluorescence also revealed the colocalization of TSPAN3 and Rab11a in the cytoplasm (Figure 5c). Furthermore, this interaction did not depend on the LEL domain of TSPAN3 (Figure 5d). Moreover, a 
dynamic change in the colocalization of TSPAN3 and Rab11a was observed at different time points after NZ washout. At 60 min after NZ washout, TSPAN3 also co-localized with Rab11a, which showed an overlapping pattern with that of the dynamic change of colocalization between TSPAN3 and $\beta 1$ integrin after NZ washout. The colocalization was maintained until 120 min after NZ washout (Figure 5e). Additionally, TSPAN3 knockdown or overexpression did not affect Rab11a levels (Additional file 10). Combining previous studies and the present findings, we hypothesized that TSPAN3 might interact with Rab11a and $\beta 1$ integrin, thereby facilitating $\beta 1$ integrin sorting into Rab11a recycling endosomes.

Immunofluorescence analysis of A549 cells at different time points after NZ washout was performed next to explore the changes in the interaction between $\beta 1$ integrin and Rab11a after TSPAN3 knockdown. The results showed that at $60 \mathrm{~min}$ after NZ washout, most of the $\beta 1$ integrin had been endocytosed in both the si-TSPAN3 and control groups. However, in the control group, the endocytosed $\beta 1$ integrin showed significant colocalization with Rab11a, whereas the colocalization between $\beta 1$ integrin and Rab11a was lower after TSPAN3 knockdown (Figure 5f, g). Correspondingly, at 120 min after NZ washout, most of the $\beta 1$ integrin in the control group had returned to the surface, whereas most of the $\beta 1$ integrin in the si-TSPAN3 group remained in the cytoplasm (Figure $5 \mathrm{~g}$ ). Moreover, immunoblotting showed that si-Rab11a transfection of both $\mathrm{A} 549$ and $\mathrm{H} 460$ cells attenuated the TSPAN3-induced elevation in $\beta 1$ integrin, p-FAK, p-MEK, p-ERK, cyclin D1, and cyclin B1 levels (Figure 5h; Additional file 11), which further supported our hypothesis. Thus, TSPAN3 promotes $\beta 1$ integrin recycling, upregulates $\beta 1$ integrin levels, and promotes NSCLC cell proliferation through a Rab11a-dependent mechanism.

\section{Discussion}

The biological function and the underlying mechanism of TSPAN3 in NSCLC have not been reported. The results of the present study show that TSPAN3 promotes $\beta 1$ integrin recycling through interaction with $\beta 1$ integrin and Rab11a, thereby upregulating $\beta 1$ integrin levels and further promoting the proliferation of NSCLC.

TSPAN3 has been reported to form a complex with $\beta 1$ integrin in oligodendrocytes and could promote the proliferation of mouse oligodendrocyte cells [17], which is consistent with the present observations in NSCLC cell lines. Recent studies have identified the key roles of some tetraspanins in the trafficking and functional regulation of membrane proteins, such as $\beta 1$ integrins [26]. In agreement with these reports, TSPAN3 was found to upregulate $\beta 1$ integrin by regulating its intracellular recycling.

Intracellular recycling of integrins greatly determines membrane integrin levels and function. The Rab11dependent route is the main pathway through which almost all integrins are transferred from the cytoplasm to the cell membrane $[27,28]$. Despite this, each integrin subunit is known to have distinct trafficking characteristics, suggesting that sophisticated mechanisms are involved in regulating the transport of specific integrins through certain routes [29]. In this study, TSPAN3 was found to be recruited to Rab11a recycling endosomes and to simultaneously accompany $\beta 1$ integrin sorting into Rab11a endosomes. Furthermore, knockdown of TSPAN3 reduced the colocalization of $\beta 1$ integrin and Rab11a 
after $\beta 1$ integrin was endocytosed. Taken together, these findings suggest that TSPAN3 functions as a sorting mediator for $\beta 1$ integrin recycling through a Rab11-dependent route. Additionally, consistently high levels of TSPAN3 were observed in NSCLC tissues and cell lines. Furthermore, high levels of TSPAN3 positively correlated with poor differentiation, lymph node involvement, advanced pathological tumormetastasis-node stage, and poor prognosis among NSCLC patients. Therefore, TSPAN3 may be a tumor promoter in NSCLC.

To our knowledge, this is the first study to report that TSPAN3 acts as a promoter of $\beta 1$ integrin intracellular recycling by facilitating $\beta 1$ integrin sorting into Rab11a recycling endosomes, which, in turn, upregulates $\beta 1$ integrin levels. These findings provide a further understanding of the mechanisms underlying integrin trafficking and provide new avenues for future exploration. For example, TSPAN3 was shown to be recruited into Rab11a endosomes, and TSPAN3 trafficking accompanies the regulation of $\beta 1$ integrin recycling; however, the mechanisms that control these processes remain unknown and would be an interesting subject for further study. The role of the LEL domain in the interaction between TSPAN3 and $\beta 1$ integrins was also herein clarified; however, the function of other TSPAN3 domains especially that of the YXXФ motif in the TM4 domain supposedly involved in vesicle sorting, remains unclear. Additionally, whether TSPAN3 regulates other aspects of $\beta 1$ integrin trafficking, such as endocytosis and lysosomal degeneration, and whether TSPAN3 regulates the recycling of other membrane receptors, such as the epidermal growth factor receptor, will have to be explored.

This study has some limitations. Herein, we report the interaction of TSPAN3 with both $\beta 1$ integrin and Rab11a; however, whether these are direct interactions was not confirmed. Further, although TSPAN3 was found to promote the colocalization of $\beta 1$ integrin and Rab11a recycling endosomes, whether this effect depends on the interaction of TSPAN3 with $\beta 1$-integrin or with Rab11a remains to be determined.

\section{Conclusions}

In summary, TSPAN3 regulates intracellular recycling of $\beta 1$ integrin via the Rab11-dependent route. This, in turn, upregulates $\beta 1$ integrin, which promotes NSCLC proliferation. To our knowledge, this is the first study to report this role of TSPAN3. Furthermore, these findings suggest that TSPAN3 is a potential target for drug development in lung cancer.

\section{List Of Abbreviations}

ATCC American Type Culture Collection

DAPI 4',6-diamidino-2-phenylindole

FBS Fetal bovine serum

FN Fibronectin 
HBE Human bronchial epithelial

IHC Immunohistochemistry

LEL Large extracellular loop

MT Microtubule

NSCLC Non-small cell lung cancer

NZ Nocodazole

OSP Oligodendrocyte-specific protein

PBS Phosphate-buffered saline

RT-PCR Real-time polymerase chain reaction

SEL Small extracellular loop

TSPAN3 Tetraspanin 3

\section{Declarations}

Ethics approval and consent to participate: The study was approved by the Medical Research Ethics Committee of the First Affiliated Hospital of China Medical University, Shenyang, China (KLS [2020] No. 2020-40-2) and was carried out in accordance with the Helsinki Declaration of 1975, as revised in 1983. The informed consent exemption was included in the ethics approval (KLS [2020] No. 2020-40-2).

Consent for publication: Not applicable

Availability of data and materials: The data that support the findings of this study are available from the corresponding author upon reasonable request.

Competing interests: The authors declare that they have no competing interests.

Funding: This work was funded by the Liaoning Province Colleges and Universities Innovation Team (LC2015029), Liaoning Provincial Education Department.

Authors' contributions: HS carried out conceptualization, methodology, validation, writing-original draft. YX carried out methodology and validation. $Y Z$ and $X J$ carried out validation. QZ and HR contributed to resources. ZZ contributed to resources and validation. $X Q$ carried out guidance and supervision. All authors were involved in writing the paper and had final approval of the submitted and published versions.

Acknowledgments: Not applicable. 


\section{References}

1. Shin S, Wolgamott L, Yoon SO. Integrin trafficking and tumor progression. Int J Cell Biol. 2012;2012:516789.

2. Caswell PT, Vadrevu S, Norman JC. Integrins: masters and slaves of endocytic transport. Nat Rev Mol Cell Biol. 2009;10:843-53.

3. Caswell P, Norman J. Endocytic transport of integrins during cell migration and invasion. Trends Cell Biol. 2008;18:257-63.

4. Pellinen T, Ivaska J. Integrin traffic. J Cell Sci. 2006;119:3723-31.

5. De Franceschi N, Hamidi H, Alanko J, Sahgal P, Ivaska J. Integrin traffic - the update. J Cell Sci. 2015;128:839-52.

6. Caswell PT, Norman JC. Integrin trafficking and the control of cell migration. Traffic. 2006;7:14-21.

7. Jones MC, Caswell PT, Norman JC. Endocytic recycling pathways: emerging regulators of cell migration. Curr Opin Cell Biol. 2006;18:549-57.

8. Moreno-Layseca P, Icha J, Hamidi H, Ivaska J. Integrin trafficking in cells and tissues. Nat Cell Biol. 2019;21:122-32.

9. Yang YG, Sari IN, Zia MF, Lee SR, Song SJ, Kwon HY. Tetraspanins: spanning from solid tumors to hematologic malignancies. Exp Hematol. 2016;44:322-8.

10. Berditchevski F, Odintsova E. Tetraspanins as regulators of protein trafficking. Traffic. 2007;8:89-96.

11. Berditchevski F. Complexes of tetraspanins with integrins: More than meets the eye. J Cell Sci. 2001;114:4143-51.

12. Fabbri M, Fumagalli L, Bossi G, Bianchi E, Bender JR, Pardi R. A tyrosine-based sorting signal in the beta2 integrin cytoplasmic domain mediates its recycling to the plasma membrane and is required for ligand-supported migration. EMBO J. 1999;18:4915-25.

13. Liu L, He B, Liu WM, Zhou D, Cox JV, Zhang XA. Tetraspanin CD151 promotes cell migration by regulating integrin trafficking. J Biol Chem. 2007;282:31631-42.

14. Shoham T, Rajapaksa R, Boucheix C, Rubinstein E, Poe JC, Tedder TF, et al. The tetraspanin CD81 regulates the expression of $\mathrm{CD} 19$ during $\mathrm{B}$ cell development in a postendoplasmic reticulum compartment. J Immunol. 2003;171:4062-72.

15. van Zelm MC, Smet J, Adams B, Mascart F, Schandené L, Janssen F, et al. CD81 gene defect in humans disrupts CD19 complex formation and leads to antibody deficiency. J Clin Invest. 2010;120:1265-74.

16. Kwon HY, Bajaj J, Ito T, Blevins A, Konuma T, Weeks $\mathrm{J}$, et al. Tetraspanin 3 is required for the development and propagation of acute myelogenous leukemia. Cell Stem Cell. 2015;17:152-64.

17. Tiwari-Woodruff SK, Kaplan R, Kornblum HI, Bronstein JM. Developmental expression of OAP1/Tspan-3, a member of the tetraspanin superfamily. J Neurosci Res. 2004;77:166-73. 
18. Tiwari-Woodruff SK, Buznikov AG, Vu TQ, Micevych PE, Chen K, Kornblum HI, et al. OSP/claudin-11 forms a complex with a novel member of the tetraspanin super family and beta 1 integrin and regulates proliferation and migration of oligodendrocytes. J Cell Biol. 2001;153:295-305.

19. Wudu M, Ren H, Hui L, Jiang J, Zhang S, Xu Y, et al. DRAM2 acts as an oncogene in non-small cell lung cancer and suppresses the expression of p53. J Exp Clin Cancer Res. 2019;38:72.

20. Nader GP, Ezratty EJ, Gundersen GG. FAK, talin and PIPKly regulate endocytosed integrin activation to polarize focal adhesion assembly. Nat Cell Biol. 2016;18:491-503.

21. Han Q, Lin X, Zhang X, Jiang G, Zhang Y, Miao Y, et al. WWC3 regulates the Wnt and Hippo pathways via Dishevelled proteins and large tumour suppressor 1 , to suppress lung cancer invasion and metastasis. J Pathol. 2017;242:435-47.

22. Jiang X, Xu Y, Ren H, Jiang J, Wudu M, Wang Q, et al. KLHL18 inhibits the proliferation, migration, and invasion of non-small cell lung cancer by inhibiting PI3K/PD-L1 axis activity. Cell Biosci. 2020;10:139.

23. Mitra SK, Schlaepfer DD. Integrin-regulated FAK-Src signaling in normal and cancer cells. Curr Opin Cell Biol. 2006;18:516-23.

24. Chao WT, Kunz J. Focal adhesion disassembly requires clathrin-dependent endocytosis of integrins. FEBS Lett. 2009;583:1337-43.

25. Ezratty EJ, Partridge MA, Gundersen GG. Microtubule-induced focal adhesion disassembly is mediated by dynamin and focal adhesion kinase. Nat Cell Biol. 2005;7:581-90.

26. Charrin S, Jouannet S, Boucheix C, Rubinstein E. Tetraspanins at a glance. J Cell Sci. 2014;127:3641-8.

27. Goldenring JR. Recycling endosomes. Curr Opin Cell Biol. 2015;35:117-22.

28. Das L, Gard JMC, Prekeris R, Nagle RB, Morrissey C, Knudsen BS, et al. Novel regulation of integrin trafficking by Rab11-FIP5 in aggressive prostate cancer. Mol Cancer Res. 2018;16:1319-31.

29. Onodera Y, Nam JM, Sabe H. Intracellular trafficking of integrins in cancer cells. Pharmacol Ther. 2013;140:1-9.

\section{Tables}

Table 1. Correlation of tetraspanin 3 (TSPAN3) expression with clinical and pathological characteristics of NSCLC patients 


\begin{tabular}{|c|c|c|c|c|c|}
\hline \multicolumn{6}{|c|}{ Correlation Parameters } \\
\hline Characteristics & $N$ & Negative & Positive & $x^{2}$ & $P$ \\
\hline Sex & & & & 0.003 & 0.958 \\
\hline Male & 51 & 32 & 19 & & \\
\hline Female & 30 & 19 & 11 & & \\
\hline Age (years $\rrbracket$ & & & & 2.009 & 0.156 \\
\hline$\leq 59$ & 38 & 27 & 11 & & \\
\hline$>59$ & 43 & 24 & 19 & & \\
\hline Histology & & & & 0.032 & 0.858 \\
\hline SCC & 28 & 18 & 10 & & \\
\hline$A C$ & 53 & 33 & 20 & & \\
\hline Differentiation & & & & 10.156 & 0.006 \\
\hline Well & 24 & 20 & 4 & & \\
\hline Moderate & 30 & 20 & 10 & & \\
\hline Poor & 27 & 11 & 16 & & \\
\hline Nodal status & & & & 7.756 & 0.006 \\
\hline No & 50 & 39 & 11 & & \\
\hline Yes & 31 & 12 & 19 & & \\
\hline TNM stage & & & & 7.063 & 0.011 \\
\hline$\nabla+\nabla$ & 64 & 45 & 19 & & \\
\hline$\nabla+\nabla$ & 17 & 6 & 11 & & \\
\hline
\end{tabular}

Table 2. Correlation between TSPAN3 expression and $\beta 1$ integrin levels in patients with non-small cell lung cancer

\begin{tabular}{|llllll|}
\hline & & \multicolumn{2}{c}{$\beta 1$ integrin } & \multicolumn{2}{c|}{ Chi-square test } \\
& & Negative & Positive & $\chi^{2}$ & $P$ \\
TSPAN3 & Negative & 5 & 2 & 6.282 & $\mathbf{0 . 0 2 2}$ \\
\cline { 2 - 5 } & Positive & 2 & 11 & & \\
\cline { 2 - 5 } & & & & & \\
\hline
\end{tabular}



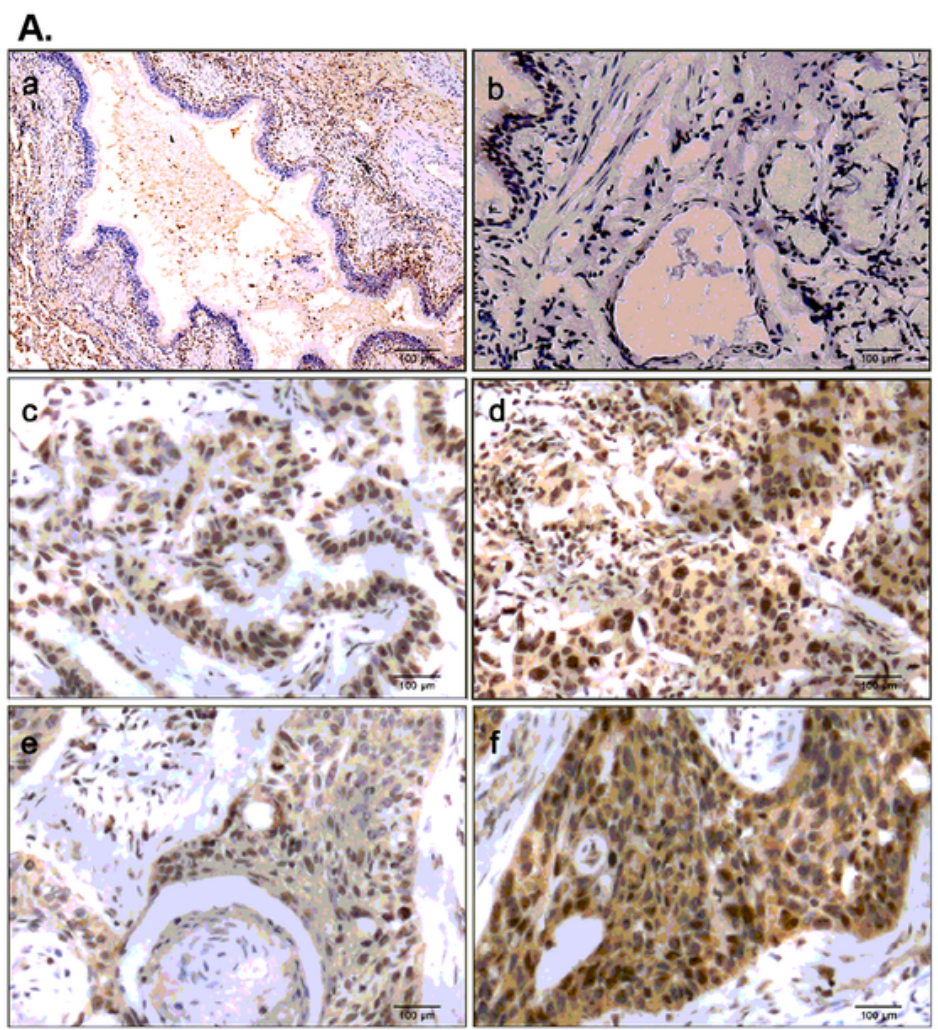

B.

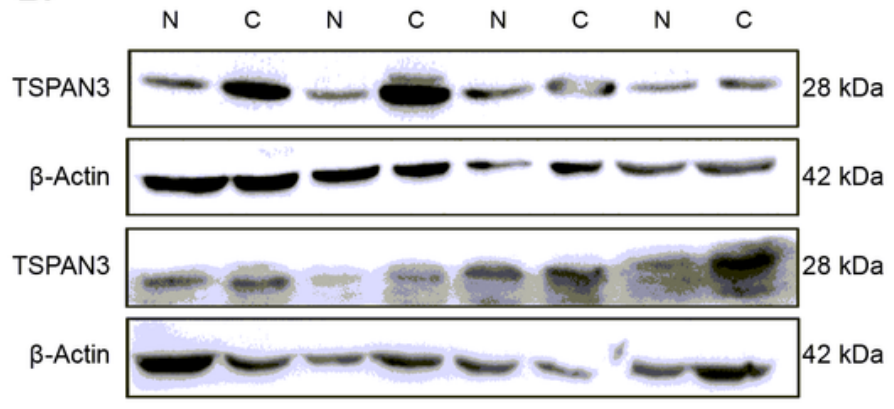

D.

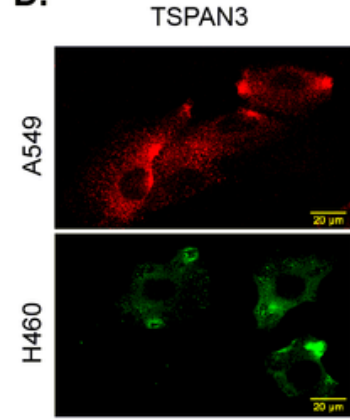

C.
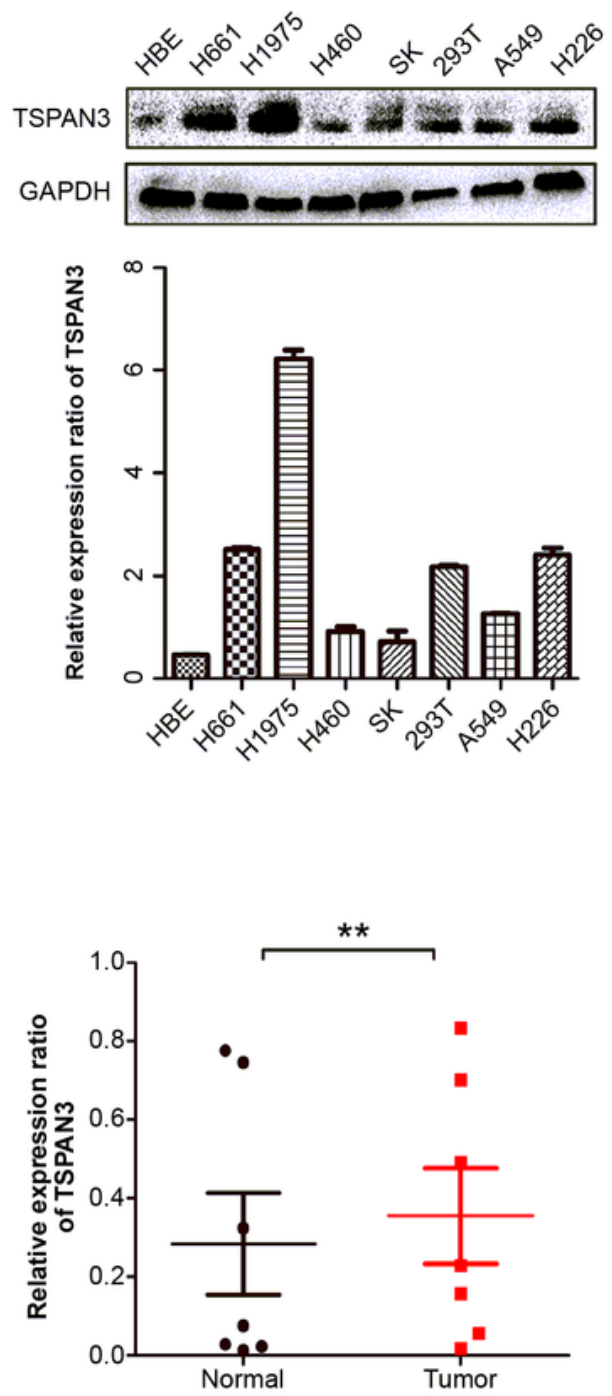

E.

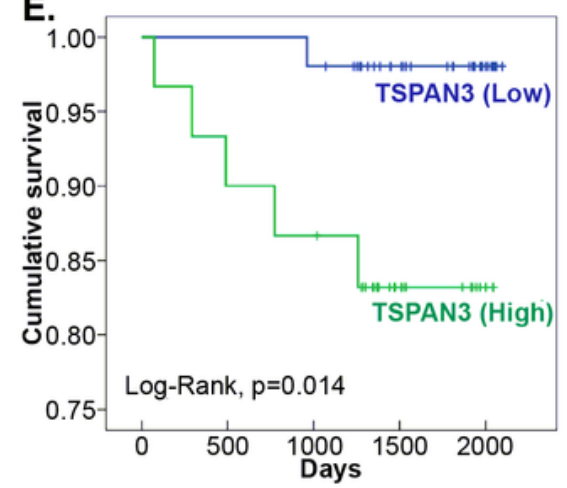

\section{Figure 1}

Expression pattern of TSPAN3 in NSCLC tissues and cell lines. (a) Tetraspanin 3 (TSPAN3) is expressed at low levels in normal bronchial epithelial cells (A) and submucosal gland cells (B) and expressed at high levels in NSCLC cells, well-differentiated adenocarcinoma (C), poorly differentiated adenocarcinoma 
(D), well-differentiated squamous carcinoma (E), and poorly differentiated squamous carcinoma (F). Magnification: $\times 400$. (b) Western blot showing that TSPAN3 levels in NSCLC tissues are higher than those in the surrounding normal tissues. $\beta$-actin served as the loading control. Relative protein expression was analyzed using Image J. ${ }^{*}<0.05$. (c) The endogenic TSPAN3 levels in six lung cancer cell lines, HBE cell line, and 297T cell line as measured by western blotting and analyzed using ImageJ; GAPDH served as the loading control ( $n=3$ independent experiments). (d) Immunofluorescence to investigate TSPAN3 localization in A549 and H460 cells. TSPAN3 is mainly localized in the cytoplasm. (e) Lung cancer patients with high expression of TSPAN3 had shorter survival periods than those with low expression of TSPAN3 $(P=0.014)$. 
A.
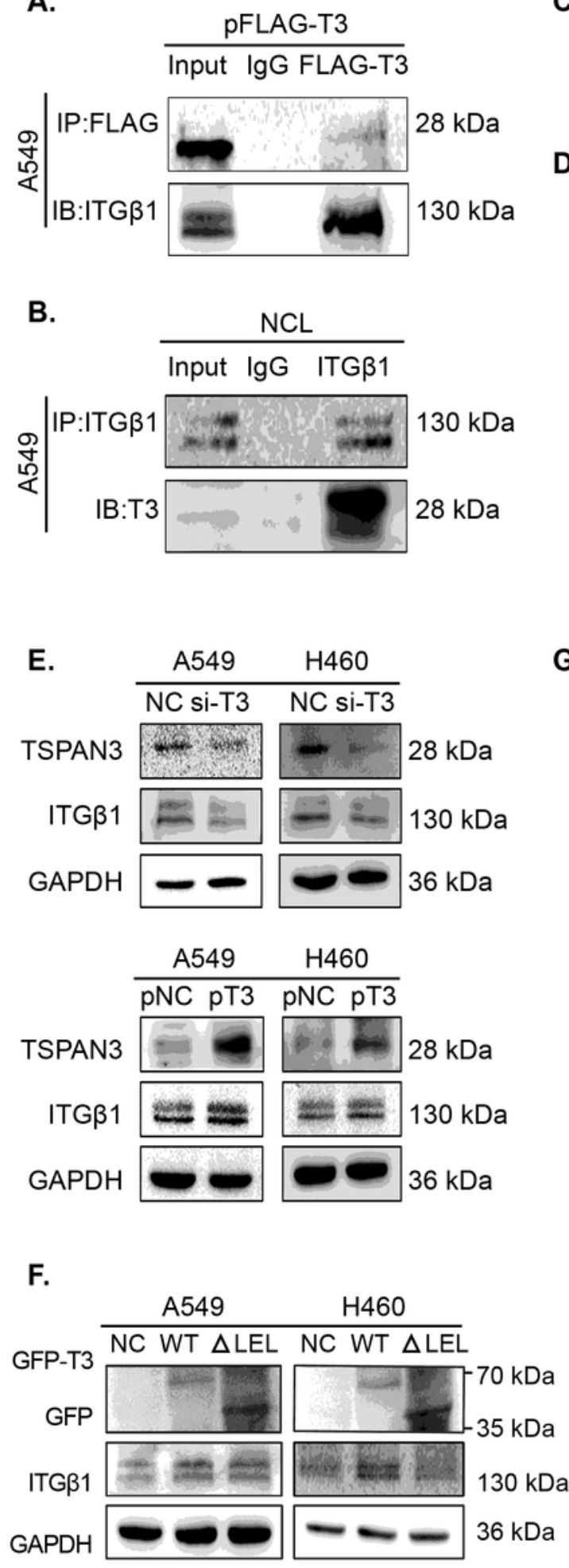

D.
C.

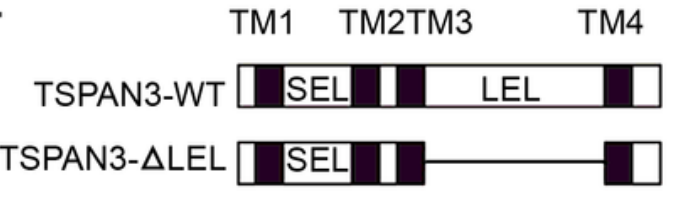

D.

A549

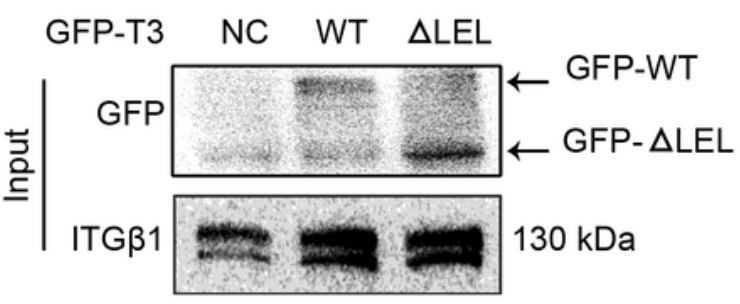

G.
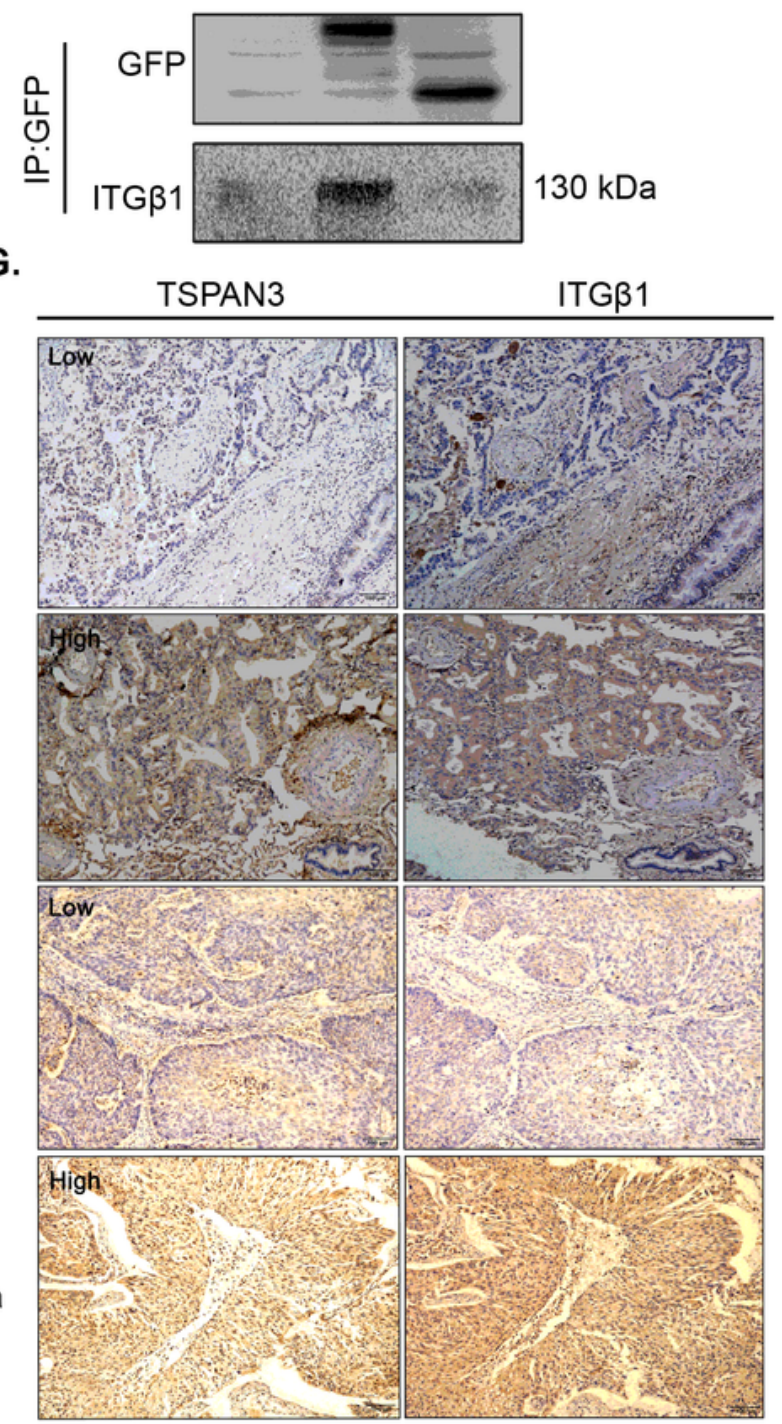

\section{Figure 2}

TSPAN3 interacts with-and upregulates- $\beta 1$ integrin. (a) Ectopically expressed tetraspanin 3 (TSPAN3/T3) interacts with $\beta 1$ integrin in A549 cells. A549 cells were transfected with the pCMV6-MycFLAG-TSPAN3 plasmid. After $48 \mathrm{~h}$ of transfection, cell lysates were immunoprecipitated with anti-FLAG antibodies or control IgG and then subjected to western blotting with anti- $\beta 1$ integrin and anti-TSPAN3 antibodies. (b) Co-immunoprecipitation revealed that endogenous TSPAN3 interacts with $\beta 1$ integrin in 
A549 cells. Cell lysates were immunoprecipitated with anti-TSPAN3 antibody or control IgG and then subjected to western blotting using anti- $\beta 1$ integrin (ITG $\beta 1$ ) and anti-TSPAN3 antibodies. (c) Schematic of TSPAN3 domains. (d) TSPAN3 interacts with $\beta 1$ integrin via the LEL domain. A549 cells were transfected with wild-type (WT) GFP-TSPAN3 or TSPAN3- $\triangle$ LEL mutant plasmids. After $48 \mathrm{~h}$ of transfection, cell lysates were immunoprecipitated with the anti-GFP antibody; the presence of $\beta 1$ integrin was measured by western blotting with the anti- $\beta 1$ integrin antibody. (e) Western blotting shows that TSPAN3 knockdown downregulated the expression of $\beta 1$ integrin in A549 and H460 cells. Overexpression of TSPAN3 had the opposite effect. GAPDH served as the loading control ( $\mathrm{n}=3$ independent experiments). (f) Overexpression of wild-type TSPAN3 resulted in upregulated expression of $\beta 1$ integrin, whereas overexpression of the TSPAN3- $\triangle$ LEL mutant did not exhibit any such effect. GAPDH served as the loading control ( $\mathrm{n}=3$ independent experiments). ( $\mathrm{g}$ ) The association between TSPAN3 and $\beta 1$ integrin was investigated using immunohistochemistry (magnification: $\times 200$ ). IB, immunoblotting; IP, immunoprecipitation. 

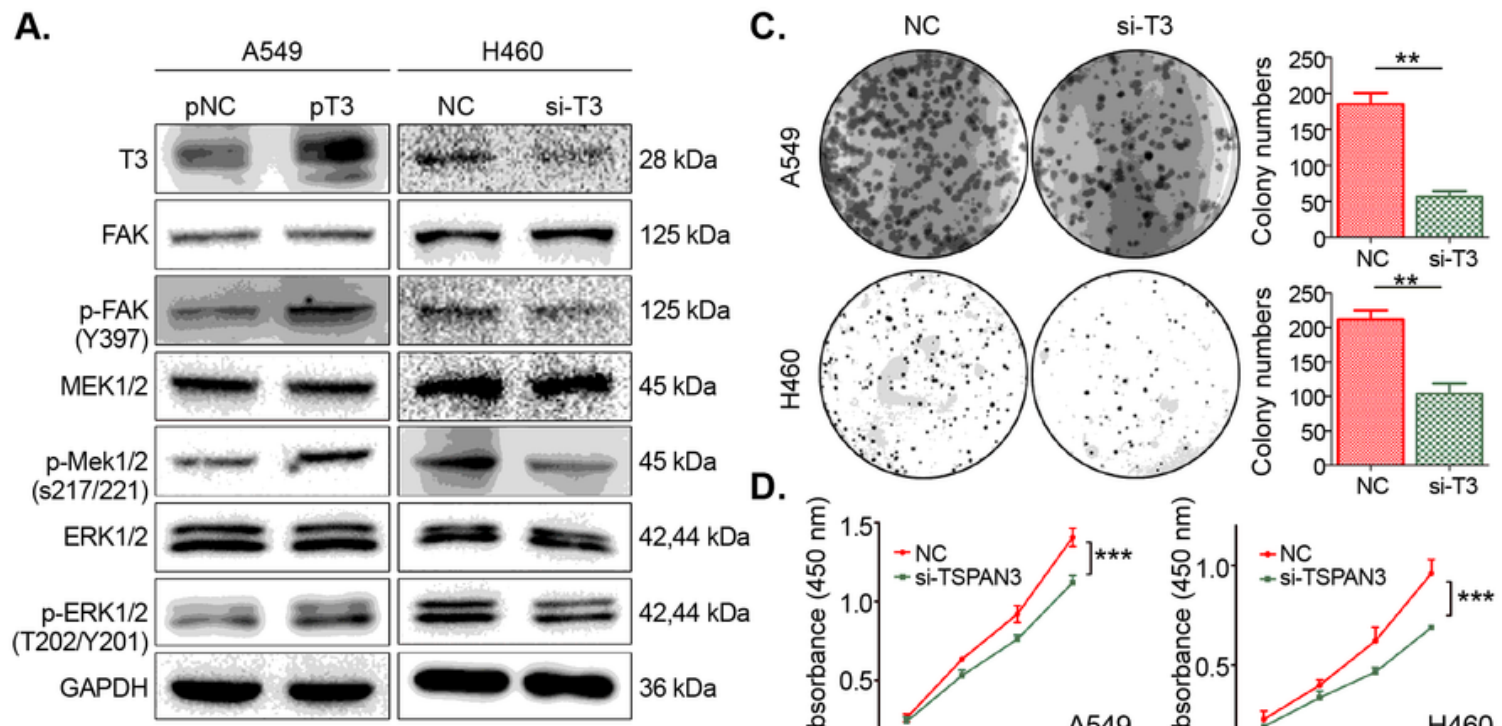

B.

E.
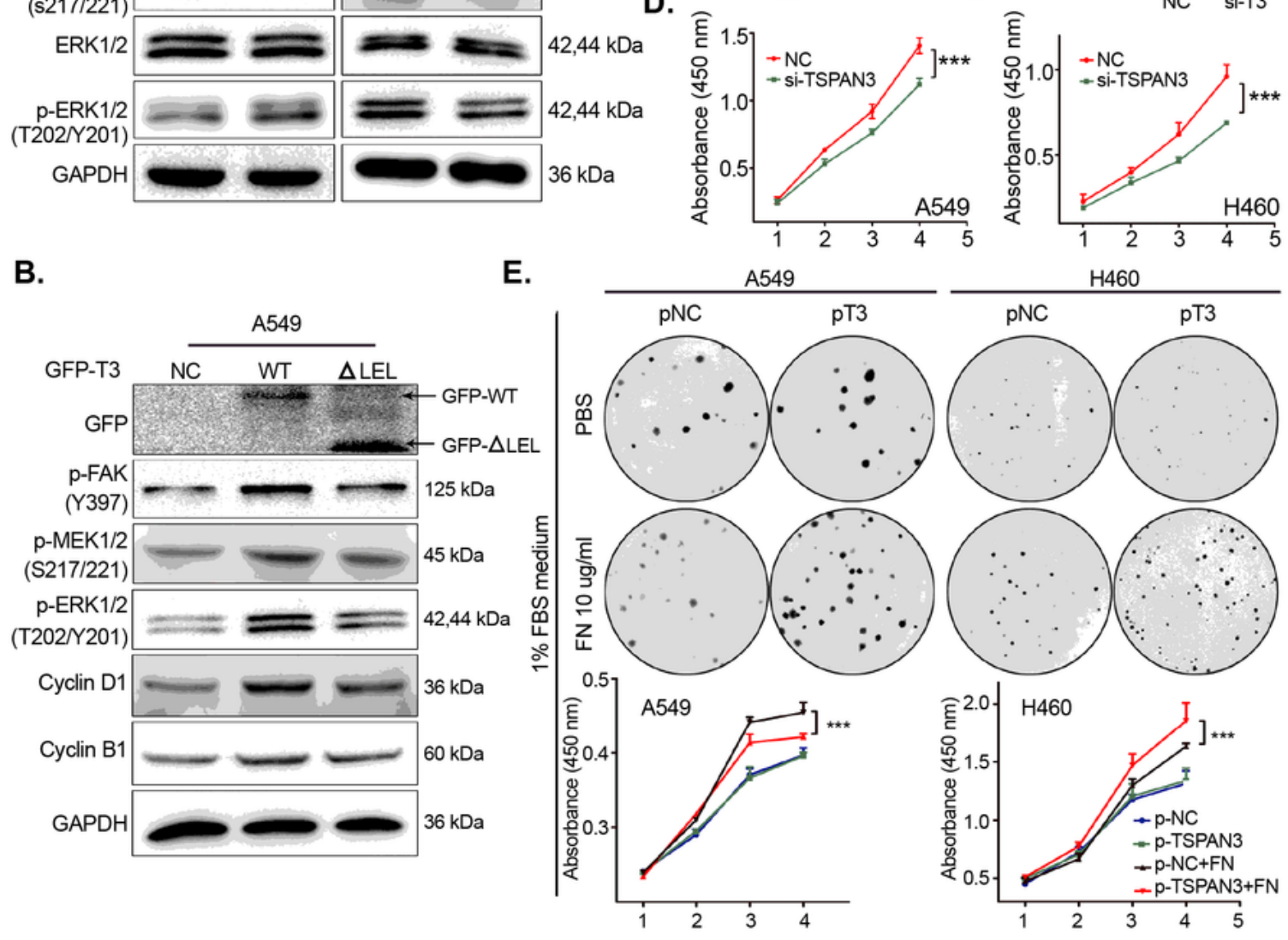

F.

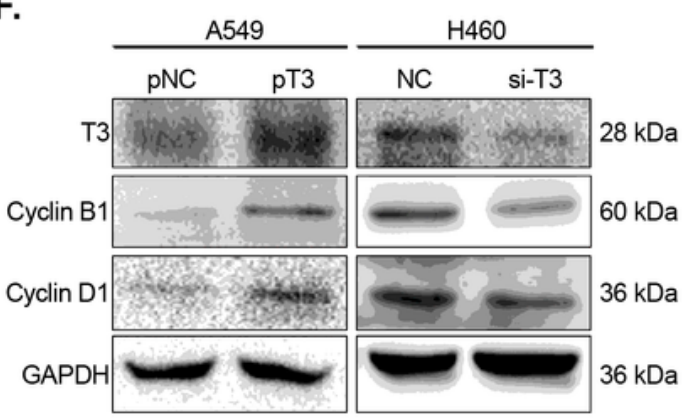

G.

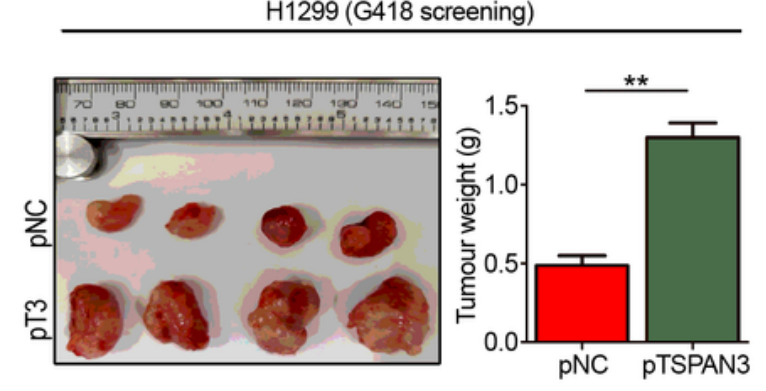

\section{Figure 3}

Effect of TSPAN3 expression on the proliferation of NSCLC cell lines. (a) Western blot showing that overexpression of tetraspanin 3 (TSPAN3/T3) in A549 cells resulted in increased phosphorylation of FAK, MEK, and ERK. Knockdown of TSPAN3 in H460 cells exhibited an opposite effect. GAPDH served as the loading control ( $\mathrm{n}=3$ independent experiments). (b) Overexpression of wild-type (WT) TSPAN3 resulted in increased phosphorylation of FAK, MEK, and ERK, and upregulation of cyclin B1 and cyclin D1. 
Overexpression of TSPAN3- $\triangle$ LEL mutants abrogated these effects. GAPDH served as the loading control ( $n=3$ independent experiments). (c, d) Colony formation and MTS assays showed inhibited proliferation of A549 and $\mathrm{H} 460$ cells in the background of TSPAN3 downregulation ( $\mathrm{n}=3$ independent experiments). (e) TSPAN3 overexpression promoted the proliferation of A549 and H460 cells in the presence of fibronectin (FN), as detected by MTS and colony formation assays ( $n=3$ independent experiments). ( $f$ ) Western blotting revealed that overexpression of TSPAN3 in A549 cells resulted in upregulated expression of cyclin B1 and cyclin D1. Opposite effects were observed upon TSPAN3 knockdown in H460 cells. GAPDH served as the loading control ( $n=3$ independent experiments). (g) The volume and weight of transplanted tumors in mice injected with cells stably overexpressing TSPAN3 were greater than those in mice injected with the empty vector (TSPAN3 vs. empty vector, volume: $0.51 \pm 0.09 \mathrm{~cm} 3$ vs. $1.38 \pm 0.13$ cm3 [P< 0.05]; weight: $0.49 \pm 0.06$ vs. $1.30 \pm 0.09$ g $[P<0.05]) . * P<0.05 ; * \star P<0.01 ; * \star * P<0.001$. FBS, fetal bovine serum; PBS, phosphate-buffered saline. 
A.

C.
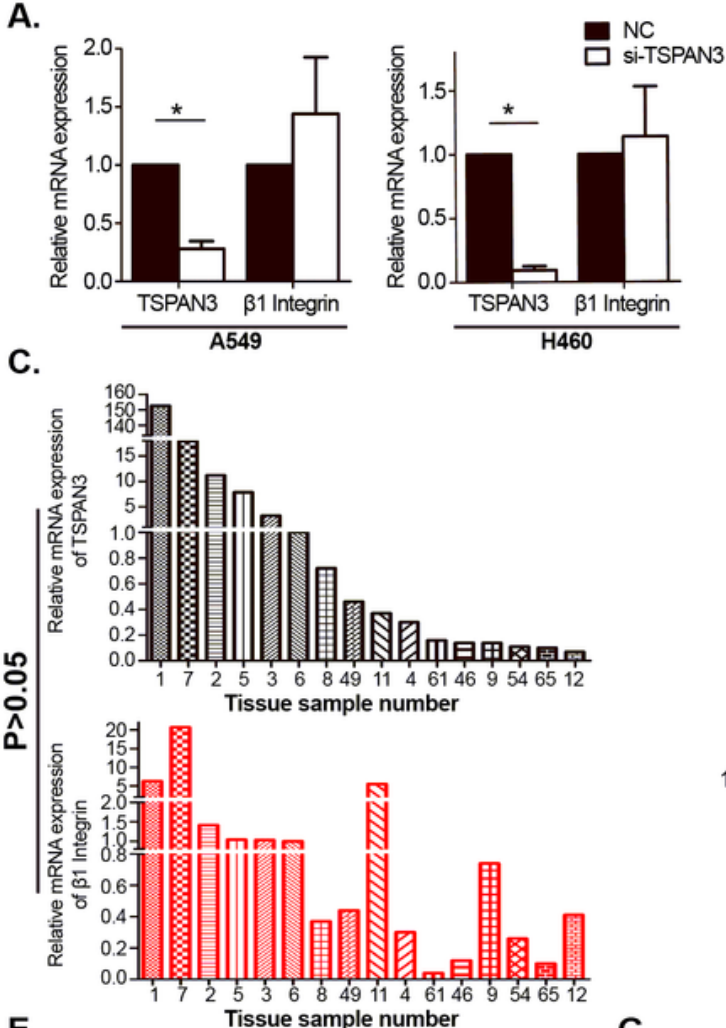

E.

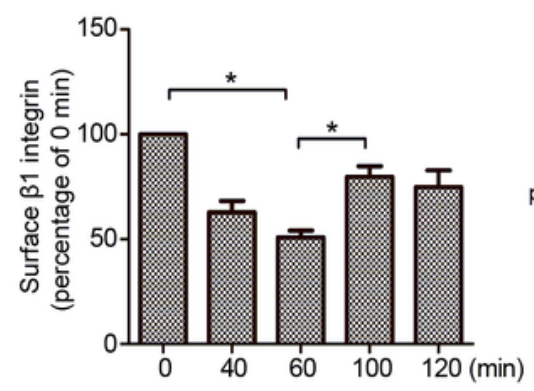

F

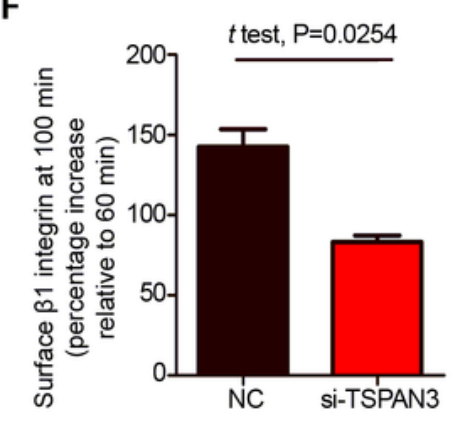

G.

H.

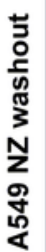

B.
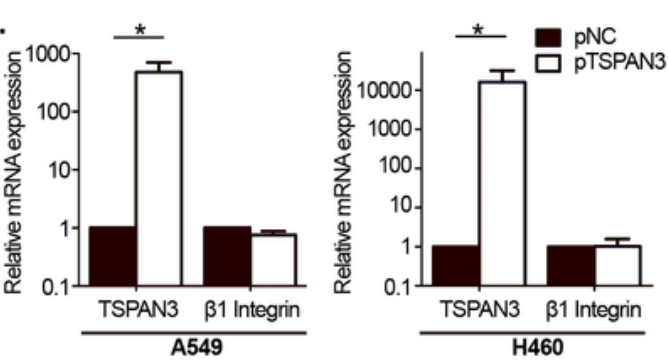

D.

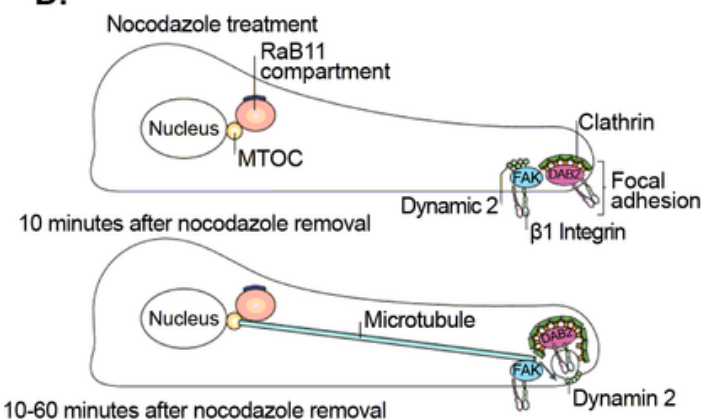

10-60 minutes after nocodazole removal
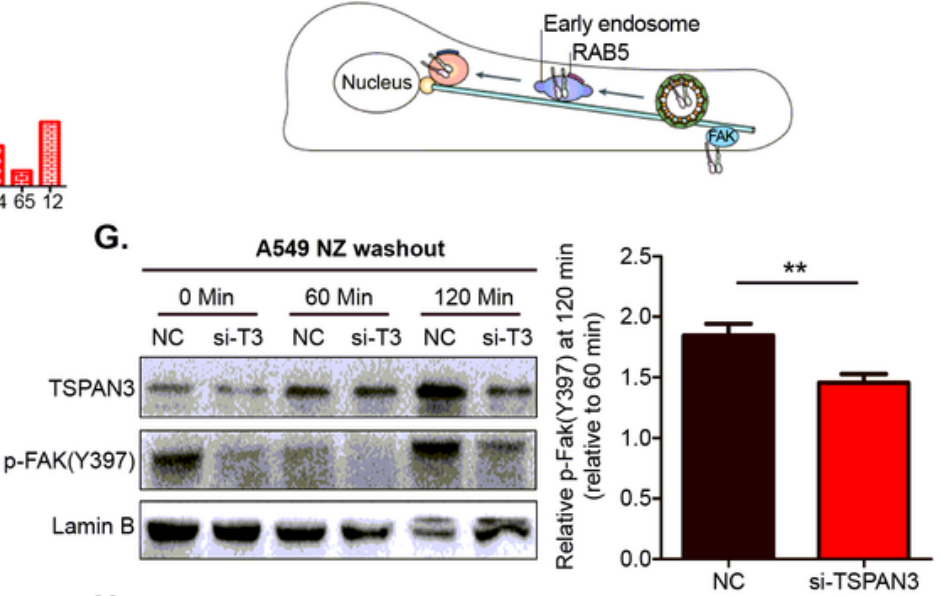

B1 Integrin

TSPAN3

Merge
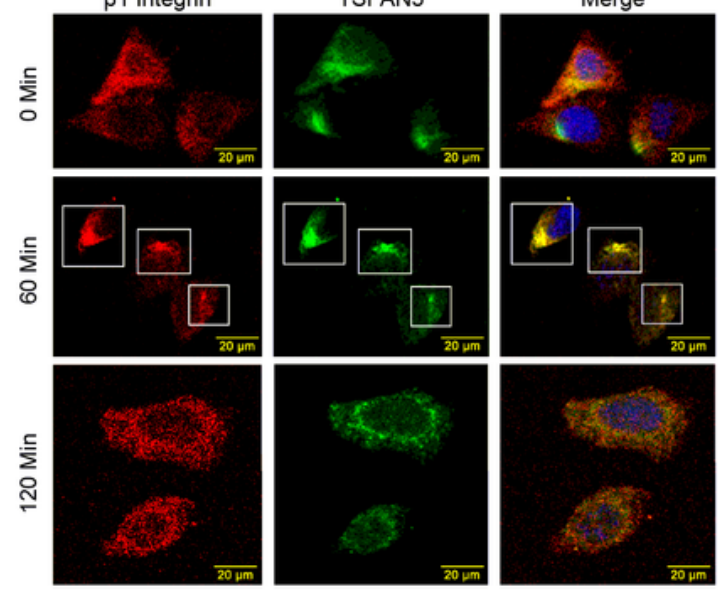

\section{Figure 4}

Impact of TSPAN3 expression on the intracellular recycling of $\beta 1$ integrin. (a) $\beta 1$ integrin (ITGB1) mRNA levels were detected through RT-PCR in A549 and H460 cells with tetraspanin 3 (TSPAN3/T3) knockdown ( $n=3$ independent experiments). (b) ITGB1 mRNA levels were detected via RT-PCR in A549 and H460 cells overexpressing TSPAN3 ( $n=3$ independent experiments). (c) Relative mRNA expression of TSPAN3 and ITGB1 in 16 NSCLC samples as analyzed by RT-PCR. The $2-\Delta \Delta$ Ct value of each sample was 
calculated with the No. 6 sample as the reference. $(P>0.05)$. (d) Model depicting the cellular phenomenon associated with nocodazole (NZ) washout [2]. (e) $\beta 1$ integrin expression on the surface of A549 cells at the indicated time points after NZ washout was assessed by flow cytometry. The expression values were normalized to those at $0 \mathrm{~min}$ ( $n=3$ independent experiments). ( $f$ ) Surface expression of $\beta 1$ integrin at $100 \mathrm{~min}$ after NZ washout relative to that at $60 \mathrm{~min}$ after NZ washout in A549 cells transfected with either si-Rab11a- or si-NC ( $n=3$ independent experiments). ( $g$ ) The levels of $p$-FAK at different time points after NZ washout. The levels of p-FAK at 100 min after NZ washout relative to that at 60 min were calculated after normalizing to Lamin $B$ levels and are shown on the right side ( $n=3$ independent experiments). (h) Immunofluorescence depicting the localization of TSPAN3 (TRITC) and $\beta 1$ integrin (FITC) in A549 cells at different time points after NZ washout. Magnification: $\times 400 .{ }^{*} P<0.05 ; * \star P<0.01$; $\star \star \star P<0.001$. 
A.

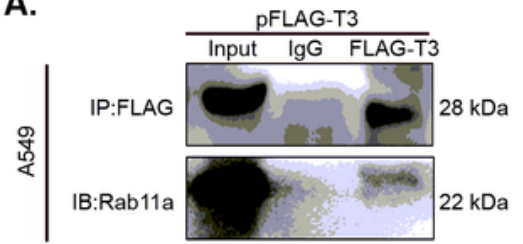

B.

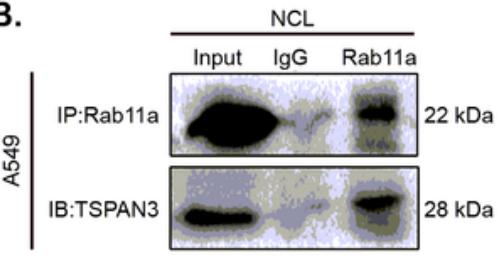

D.

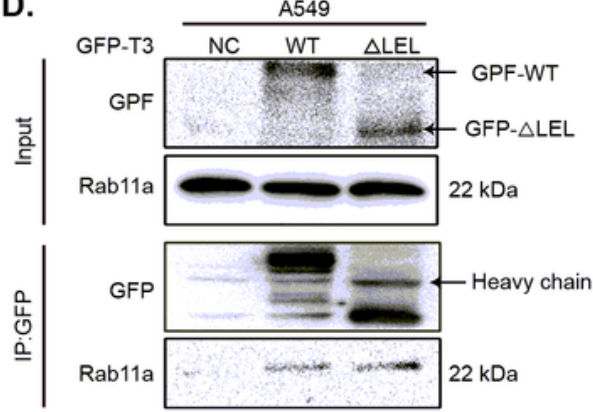

F.

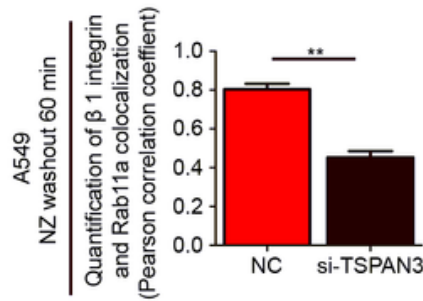

H.

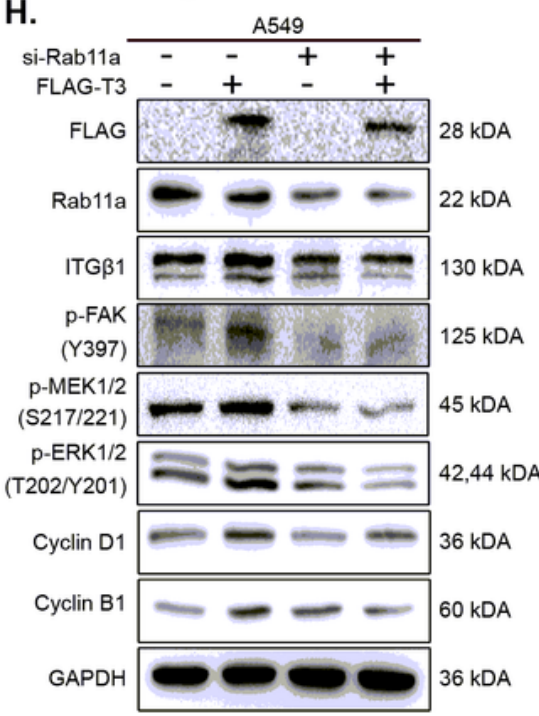

c.

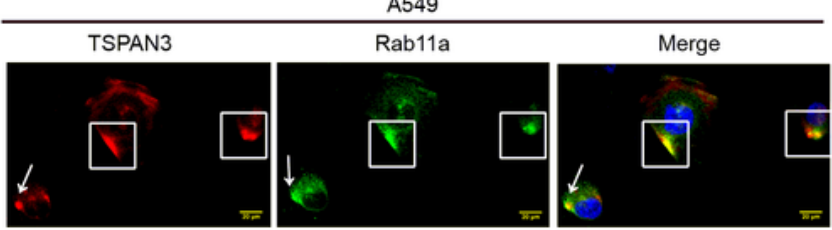

E.

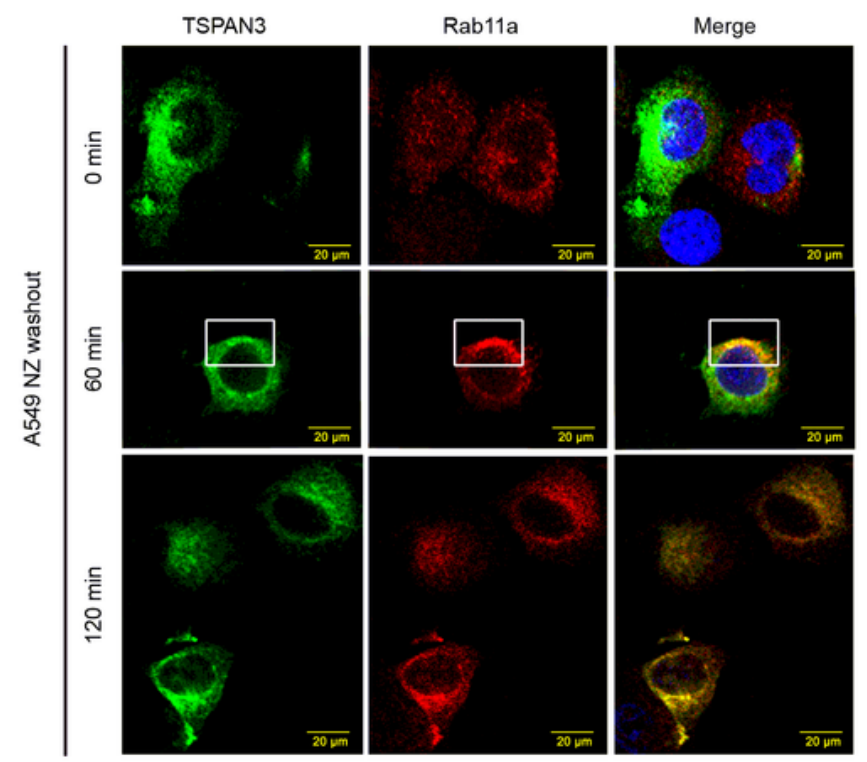

G.

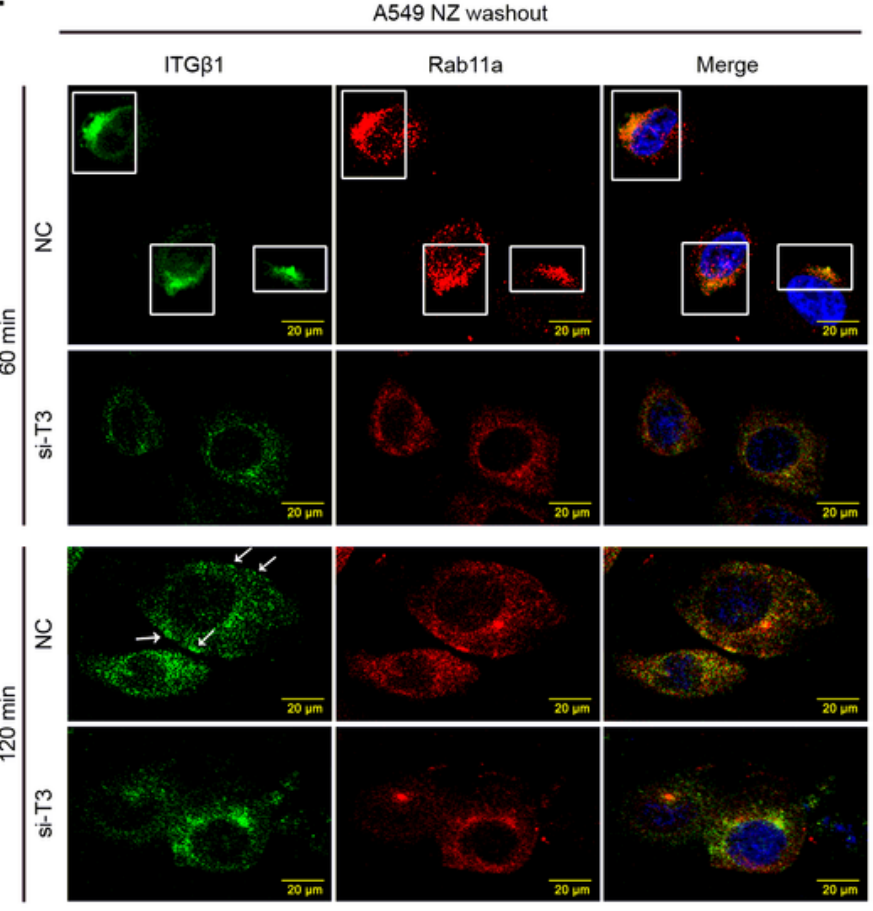

Figure 5

Effect of TSPAN3 on $\beta 1$ integrin recycling. (a) Co-immunoprecipitation assays showing the interaction between exogenous tetraspanin 3 (TSPAN3/T3) and endogenous Rab11a in A549 cells. Forty-eight hours after transfecting A549 cells with pCMV6-Myc-FLAG-TSPAN3, cell lysates were immunoprecipitated with anti-FLAG antibodies or control IgG and subjected to western blotting with anti-Rab11a and anti-FLAG antibodies. (b) Co-immunoprecipitation assay showing the interaction between endogenous TSPAN3 and 
Rab11a in A549 cells. Cell lysates were immunoprecipitated with anti-TSPAN3 antibody or control IgG and subjected to western blotting with anti-Rab11a and anti-TSPAN3 antibodies. (c) Immunofluorescence showing the localization of TSPAN3 (TRITC) with Rab11a (FITC) in the cytoplasm of A549 cells. Magnification: $\times 400$. (d) Western blot indicating that TSPAN3-Rab11a interaction does not depend on the LEL domain of TSPAN3. A549 cells were transfected with plasmids encoding wild-type GFP-TSPAN3 or TSPAN3 mutant. Forty-eight hours after transfection, cell lysates were immunoprecipitated with the anti-GFP antibody; the presence of Rab11a in the anti-GFP-precipitated complex was examined through western blotting with the anti- Rab11a antibody. (e) Immunofluorescence showing the colocalization of TSPAN3 (TRITC) and Rab11a (FITC) in A549 cells at different time points after nocodazole (NZ) washout (magnification: $\times 400)$. (f) Pearson correlation coefficient of the colocalization between Rab11a and $\beta 1$ integrin at 60 min after NZ washout. (g) Immunofluorescence showed that TSPAN3 knockdown resulted in reduced colocalization of $\beta 1$ integrin and Rab11a (60 min after NZ washout) and the surface expression of $\beta 1$ integrin (120 min; indicated by arrowheads). A549 cells transfected with TSPAN3 or NC siRNA for $48 \mathrm{~h}$ were analyzed to investigate colocalization between endogenous $\beta 1$ integrin and Rab11a at different time points after NZ washout through confocal microscopy (magnification: $\times 400)$. (h) Western blot indicating that Rab11a knockdown attenuated the TSPAN3-induced increase in $\beta 1$ integrin, p-FAK (Tyr397), p-MEK, p-ERK, cyclin B1, and cyclin D1 expression. GAPDH served as the loading control ( $n=3$ independent experiments).

\section{Supplementary Files}

This is a list of supplementary files associated with this preprint. Click to download.

- Additionalfile1and2.docx

- Additionalfile3.tif

- Additionalfile4.tif

- Additionalfile5.tif

- Additionalfile6.tif

- Additionalfile7.tif

- Additionalfile8.tif

- Additionalfile9.tif

- Additionalfile10.tif 